\title{
Enfermagem enQuanto disciplina: Que campo de conhecimento identifica a profissão?
}

\author{
Nursing as a discipline: what scientific knowledge field identifies the profession?
}

\section{Enfermería en cuanto disciplina: Qué campo del conocimiento la identifica como profesión?}

\author{
Maria Cecília Puntel de Almeida', Silvana Martins Mishima', Maria José Bistafa Pereira', \\ Pedro Fredemir Palha', Tereza Cristina Scatena Villa', Cinira Magali Fortuna', Sílvia Matumoto' \\ 'Universidade de São Paulo. Escola de Enfermagem de Ribeirão Preto. \\ Departamento de Enfermagem Materno-Infantil. Ribeirão Preto, SP
}

Submissão: 12/12/2008

Aprovação: 30/07/2009

\begin{abstract}
RESUMO
Propõe-se uma reflexão crítica acerca do conhecimento Que vem sendo produzido pela enfermagem, Que a identifica como uma disciplina e a suficiência ou não deste conhecimento, pautado nas ciências positivas e, portanto, com arcabouço de ciência e disciplina científica para constituir os fundamentos do Cuidado, o objeto de trabalho da prática de enfermagem. Numa perspectiva histórica aborda como a enfermagem brasileira, principalmente, a partir da década de 90 do último século, vem se dedicando a problematização teórico-prática e em experiências concretas sobre o Cuidado ancorado nas diversas vertentes do conhecimento, sinalizando a necessidade da continuidade dessa produção de conhecimento Que se articule às experiências práticas/cotidianas da enfermagem, incluindo para tal outras correntes do conhecimento voltadas para a dimensão ontológica, existencial, dialógica e outras, pois o processo saúde-doença é sempre complexo carregado de valores éticos Que expressa a singularidade do homem.
\end{abstract}

Descritores: Enfermagem; Conhecimentos, atitudes e práticas em saúde; Força de trabalho, Teoria de enfermagem; PesQuisa em enfermagem.

\begin{abstract}
A critical reflection is proposed on the knowledge that has been produced by Nursing, that identifies it as a discipline, and on whether that knowledge is enough, based on the positive sciences and therefore with a framework of science and scientific discipline in order to build the fundamentals of Care, which is the object with which Nursing works. Under a historical perspective, this paper analyzes how Brazilian Nursing, especially since the 1990s, has been dedicating itself to theoretical and practical problems and to concrete experiences on Care anchored to various knowledge dimensions, signalizing the need for continuity of knowledge production that is linked to practical and routine experiences of Nursing, including other knowledge approaches that are focused on ontological, existential, dialogical and other dimensions, because the health-disease process is always complex and filled with ethical values which express the singularity of men.

Descriptors: Nursing; Health knowledge, attitudes and practice; Labor force; Nursing theory; Nursing research.
\end{abstract}

\section{RESUMEN}

Se propone una reflexión crítica sobre el conocimiento que ha sido producido por la enfermería, Que la identifica como una disciplina, y sobre la suficiencia o no de ese conocimiento, basado en las ciencias positivas y, por lo tanto, con base de ciencia y disciplina científica para construir los fundamentos del Cuidado, Que es el objeto de trabajo de la práctica de enfermería. Desde una perspectiva histórica, se aborda cómo la enfermería brasileña, principalmente a partir de la década de 1990, viene dedicándose a la problematización teóricopráctica y a experiencias concretas sobre el Cuidado ancorado en las diversas vertientes del conocimiento, indicando la necesidad de continuidad de esa producción de conocimiento Que se articule con las experiencias prácticas/cotidianas de la enfermería, incluyendo, para tal, otras corrientes del conocimiento vuelto hacia la dimensión ontológica, existencial, dialógica y otras, pues el proceso saludenfermedad es siempre complejo y cargado de valores éticos, lo que expresa la singularidad del hombre.

Descriptores: Enfermería; Conocimientos, actitudes y prácticas en Salud; Fuerza de trabajo; Teoría de enfermería; Investigación en Enfermería. 


\section{INTRODUÇÃO}

Estamos na primeira década do século XXI e constatamos Que a área da saúde convive com grandes paradoxos. De um lado houve intensa incorporação de tecnologias com o desenvolvimento de aparelhagens sofisticadas Que utilizam o raio laser para intervenções, tecnologias tridimensionais de imagens para diagnóstico, ultrasonografias, ressonâncias magnéticas, desenvolvimento de pesQuisas relacionadas ao projeto genoma, pesquisas com drogas farmacológicas para a terapia de cânceres, AIDS e outras doenças, desenvolvimento de vacinas para proteção de crianças e adultos contra considerável número de doenças e a erradicação de algumas outras.

Estes são alguns dentre muitos outros avanços tecnológicos voltados para a saúde. Apesar deste avanço, estamos convivendo com outros problemas na área da saúde como as doenças reemergentes, dentre elas a tuberculose, cólera, febre amarela e ainda aQuelas Que são endêmicas em muitas regiões do mundo, como por exemplo, a dengue.

As condições crônicas se colocam também como um grande desafio dos nossos dias, como a alta incidência e prevalência de doenças como o diabetes, a hipertensão, a obesidade, a doença mental e outras, sem deixar de falar sobre a violência e o uso abusivo de álcool e drogas e a desigualdade social. Outro paradoxo é que a medicina tecnológica, Que se desdobra em muitas especialidades nas últimas décadas, não tem conseguido por si só dar conta da ampla e profunda dimensão e complexidade do processo saúdedoença-cuidado.

Merhy ${ }^{(1)}$ diz Que não é da ausência do aparato tecnológico Que os usuários reclamam Quando acessam os serviços de saúde, mas da incapacidade expressa na desatenção, descuido, descaso dos trabalhadores de saúde em dar ouvidos e atenção às suas dores e sofrimentos. Ayres ${ }^{(2)}$ reforça esta posição dizendo Que só a lógica da racionalidade técnica não é suficiente para produzir o Cuidado em saúde.

No período de meados dos anos 70 até os anos 90, participamos no Brasil de intensa mobilização política visando transformações no sistema de saúde brasileiro. Conseguimos em 1988, colocar na Constituição brasileira o capítulo da saúde, Que veio sendo preparado e consensuado desde a $8^{\text {a }}$ Conferência Nacional de Saúde em 1986. Assim, foi criado o Sistema único de Saúde, considerando a saúde como um direito universal, gratuito, Que garantisse a integralidade e eue fosse de eualidade para todos os brasileiros. O sistema privado foi definido como complementar subordinado e regulado pelo SUS.

O Sistema devia ser descentralizado e os estados e municípios deveriam assumir a responsabilidade pelas ações de forma coordenada e autônoma, com financiamento do governo federal Que deveria destinar parte de seu orçamento para a saúde. Em 1990, consegue-se sancionar as Leis Orgânicas da Saúde - Leis $n^{\circ} 8080 / 1990$ e no $8142 / 1990$, Que passam a regulamentar a operacionalização do setor, juntamente com as Normas Operacionais Básicas. Esta mobilização política e legislação na saúde provocaram mudanças incontestáveis, mas ainda não totalmente suficientes.

O Ministério da Saúde, os profissionais, usuários e entidades representativas do setor saúde continuaram discutindo a necessidade de se pensar e organizar um novo modelo assistencial de saúde Que de fato colocasse em operação os princípios do SUS, e Que não fosse centrado exclusivamente na doença, e no profissional médico, priorizando só os aspectos individuais e hospitalocêntricos. Assim, é neste final do século XX e início do XXI que são propostas outras lógicas e modos de produzir saúde. Surgem várias estratégias como a Saúde da Família, a Integralidade da Atenção, a Humanização da Saúde, Promoção da Saúde e outras alternativas de modelos de saúde, usuários centrados, voltados todos eles para promover a vida e a Qualidade de vida.

Em 2006 resgatam-se os princípios da Atenção Primária de Saúde por meio da Política Nacional de Atenção Básica ${ }^{(3)}$ Que se orienta pelos princípios da universalidade, acessibilidade, coordenação do cuidado, do vínculo e continuidade, da integralidade, responsabilização, humanização, equidade e participação social. Mas, no nosso "Que fazer" cotidiano, continuamos insatisfeitos com o modo Que estamos produzindo saúde e também, os usuários reclamam sobre a assistência Que recebem.

Diante deste Quadro, nos propomos a fazer uma reflexão crítica e propiciar uma discussão sobre, Qual é o conhecimento Que vem sendo produzido pela enfermagem, Que a identifica como uma disciplina e se este conhecimento tem sido suficiente e adequado para constituir os fundamentos do Cuidado, Que é o objeto de trabalho da prática de enfermagem.

Outra pergunta, Que é um desdobramento da anterior, é se o conhecimento científico da enfermagem pautado nas ciências positivas, e, portanto, com arcabouço de ciência e disciplina científica Qualifica a assistência de enfermagem, no sentido da produção Cuidado.

\section{O CONHECIMENTO TÉCNICO-CIENTÍFICO EM ENFERMAGEM}

Tratar do conhecimento em saúde nos remete ao campo das ciências, tecnologias e práticas, claro Que sem deixar de mencionar o campo das políticas públicas. Portanto, a organização destas atividades de saúde, segundo Ayres ${ }^{(2)}$, é orientada pela racionalidade instrumental, baseada nas ciências empírico-analíticas. A validação das tecnologias ocorre Quando estas produzem o efeito esperado, ou melhor, o êxito técnico da ação. A enfermagem, enquanto um campo de saberes e práticas vem ao longo do século $\mathrm{XX}$ construindo, acumulativamente, seu conhecimento e produzindo historicamente suas práticas.

Almeida ${ }^{(4)}$ em um estudo histórico-social realizado sobre o saber de enfermagem desde a emergência da profissão em Londres, a partir de 1860, até os anos 80 do último século, resgata o conhecimento deste período histórico. Os Estados Unidos, a partir das primeiras décadas do século XX, passa a liderar a construção do conhecimento influenciado pelo Relatório Flexner Que passa a disciplinar o conhecimento científico da prática médica.

Almeida ${ }^{(4)}$ considera Que as técnicas foram a primeira expressão do saber de enfermagem organizadas nas primeiras décadas do último século, para dar conta do aumento crescente dos cuidados de enfermagem, devido ao grande número de internações e ao aumento de ações Que, consideradas manuais, passavam das mãos dos médicos para as enfermeiras e, posteriormente, para o pessoal 
auxiliar. A divisão técnica do trabalho acelerou este processo, na organização dos hospitais, principalmente nos Estados Unidos da América.

Na década de 50, surge também nos Estados Unidos da América, a preocupação em organizar os princípios científicos da prática profissional. Até então a enfermagem era vista como não científica e suas ações baseadas na intuição. Foi organizado um estudo na Escola de Enfermagem da Universidade de Washington que teve a duração de cinco anos e encabeçado por comitês de especialistas, um de ciências naturais e um de ciências sociais, juntamente com especialistas em física e Química. Assim, o conhecimento de enfermagem, na década de 50, procura delinear-se buscando uma fundamentação para as técnicas de enfermagem, e esta é chamada científica e tem suas bases nas ciências naturais (anatomia, microbiologia, fisiologia, patologia) e também nas ciências sociais. Assim, busca-se a cientificidade da profissão na aproximação com a medicina, e conseQüentemente com sua autoridade.

Os conceitos e definições de enfermagem de Vírginia Henderson, na década de 50, têm também como fundamentação os princípios científicos. A autora deixa claro que a enfermeira é a "autoridade do cuidado básico" de enfermagem, sua "única função" na Qual ela trabalha independente, e os componentes inerentes a este cuidado, Que são as funções da vida física, psíquica e social. Henderson, ${ }^{(5)}$ define Que "a função peculiar da enfermeira é dar assistência ao indivíduo doente ou sadio no desempenho de atividades Que contribuem para manter a saúde ou para recuperála (ou ter uma morte serena) - atividades que ele desempenharia só, se tivesse a força, vontade ou o conhecimento necessários. É fazê-lo de modo que o ajude a ganhar sua independência o mais rápido possível”.

$\mathrm{Na}$ continuidade do estudo, Almeida ${ }^{(4)}$ diz que o saber expresso pelos princípios científicos nas décadas de 50 e 60, passa também a ser visto pela liderança da enfermagem como dependente, sem possuir uma natureza específica e sem ser autônomo. Surge uma discussão ${ }^{(6)}$ para conceituar a enfermagem como ciência, Que é definida como "um corpo de conhecimentos científicos acumulativos Que é trazido da física, biologia e ciências do comportamento e Que, pelo processo de síntese, torna-se unicamente enfermagem.

O instrumental construído para orientar a enfermagem, na busca de sua autonomia, como campo específico de saber, foi as teorias de enfermagem, permitindo uma delimitação de seus limites de atuação, no trabalho com outros profissionais. Todos nós conhecemos a contribuição das teoristas norte-americanas e dentre elas destacamos, Sister Callista Roy ${ }^{(7,8)}$, Myra Estrin Levine ${ }^{(9)}$, Dagmar Brodt $^{(10)}$, Martha Rogers ${ }^{(1)}$, Imogene King ${ }^{(12)}$, Elizabeth D. Oren ${ }^{(13)}$.

Não podíamos deixar de mencionar a primeira enfermeira brasileira Que criou o modelo conceitual das necessidades humanas básicas, em 1979, Wanda de Aguiar Horta ${ }^{(14)}$. Este enfoque da construção do corpo de conhecimentos específicos da enfermagem vem sendo expresso por uma terminologia variada como: a natureza específica da enfermagem, a formalização dos conceitos e teorias, a construção de marcos teórico de referência, de modelos, etc.

Neste mesmo movimento do surgimento das teorias de enfermagem, é introduzido também nos Estados Unidos da América o processo de enfermagem Que nos anos 70 ampliou as fases do mesmo Que foram definidas como assessment, diagnóstico, planejamento, implementação e avaliação ${ }^{(15)}$.
No Brasil, o Conselho Federal de Enfermagem, através da Resolução 272/2002(16) estabelece Que a Sistematização da Assistência de Enfermagem - SAE - é uma atividade privativa do enfermeiro, utiliza método e estratégia de trabalho científico para a identificação das situações de saúde/doença, subsidiando ações de assistência de Enfermagem Que possam contribuir para a promoção, prevenção, recuperação e reabilitação da saúde, do indivíduo, família e comunidade.

Define ainda a implementação da SAE deve ocorrer em toda instituição de saúde, pública e privada. Este é composto por seis etapas: Histórico de Enfermagem, Exame Físico, Diagnóstico de Enfermagem, Prescrição da Assistência de Enfermagem, Evolução da Assistência de Enfermagem e Relatório de Enfermagem. Portanto, o Processo de Enfermagem (PE) ou Sistematização da Assistência de Enfermagem (SAE) possibilita o emprego das fases do método científico e é uma atividade exclusiva do enfermeiro.

É no movimento do processo de enfermagem Que se constrói os Sistemas de Classificação dos problemas de enfermagem. O primeiro sistema foi o de classificação dos diagnósticos de enfermagem conhecido como NANDA - North American Nursing Diagnosis Association, Que teve seu ponto de partida na Primeira Conferência do Grupo Norte-Americano para Classificação dos diagnósticos de enfermagem, em 1973.

Em 1982, a NANDA foi formalmente organizada ${ }^{(17)}$. Cruz ${ }^{(18)}$ considera Que os diagnósticos de enfermagem são: "indicadores de necessidades de cuidados de enfermagem da pessoa ou grupo Que está sendo cuidado. Partindo do pressuposto que o cuidar é a nossa clínica, as necessidades de cuidados de enfermagem possíveis compõem uma constelação de focos aos Quais deve se dirigir o desenvolvimento articulado de um corpo de conhecimento".

Assim, também no final da década de 80, o Conselho Internacional de Enfermagem (ICN) iniciou estudos objetivando a elaboração de um sistema Que descrevesse a prática de enfermagem a partir de uma nomenclatura compartilhada pelas enfermeiras de todo o mundo. Este se denomina Classificação Internacional para a Prática de Enfermagem - ICNP - International Classification for Nursing Practice. Classifica os fenômenos de enfermagem, as intervenções e os resultados.

Dentre seus objetivos destacamos: "estabelece uma linguagem comum para descrever a prática de enfermagem, para promover a comunicação entre enfermeiras e entre estas e outros profissionais; descrever os cuidados de enfermagem; possibilitar a comparação de dados de enfermagem; demonstrar ou projetar tendências para provisórios tratamentos de enfermagem e cuidados e alocar recursos para os pacientes, de acordo com suas necessidades baseadas no diagnóstico de enfermagem e promover dados, informações sobre a prática de enfermagem influenciando as políticas"(19).

A partir da CIPE, foi realizado no Brasil, pela Associação Brasileira de Enfermagem a CIPESC - Classificação Internacional da Prática de Enfermagem em Saúde Coletiva, no final dos anos 90. O Projeto brasileiro CIPESC, além de seguir os fundamentos e a areuitetura da CIPE, traz no seu arcabouço teórico, duas vertentes conceituais Que lhe dão sustentação, a saúde coletiva e o processo de trabalho em saúde ${ }^{(19)}$.

Em 1987, é lançada nos Estados Unidos a Classificação das Intervenções de Enfermagem - NIC Que é uma taxonomia Que inclui as atividades de enfermagem executadas. Resultou de um 
grande projeto de um grupo de pesquisadores da Faculdade de Enfermagem da Universidade de lowa ${ }^{(20)}$.

Conclui-se Que as lideranças da enfermagem vieram construindo este corpo de conhecimentos específicos para garantir a identidade da profissão, a sua autonomia, autoridade e responsabilidade, enfim dar à enfermagem o estatuto de disciplina e de ciência aplicada na área da saúde, para garantir o Cuidado Qualificado.

Cabe destacar Que o desenvolvimento da prática de enfermagem e de um corpo de conhecimentos Que possibilite sua sustentação não ocorre em cada um destes momentos de forma desarticulada da organização mais geral da sociedade, da compreensão de homem, de verdade, de ciência, do processo saúde-doença-cuidado e da conformação das práticas de saúde. Assim, a construção do conhecimento de enfermagem encontra-se marcado pelas determinações histórico-sociais presentes.

\section{A CONTRIBUIÇÃO DA FILOSOFIA HERMENÊUTICA PARA PRODUZIR O CUIDADO EM SAÚDE/ENFERMAGEM}

Mesmo que consideremos a enfermagem como prática social e, portanto, como trabalho, não negamos o cientificismo da profissão, consideramos Que este corpo de conhecimentos específico é Que possibilita a ela ser uma profissão e como tal prestar, serviços legitimados socialmente.

O Que se Quer discutir nesta fala é suscitar o debate no sentido de considerar que este conhecimento produz sim o êxito técnico, Que é o resultado esperado das ciências empírico-analíticas, Que se baseiam na verificação lógica e/ou experimental de relações de necessidade entre proposições, com vistas à apreensão de relações de caráter causal ${ }^{(2)}$. Mas, nas práticas de saúde e de enfermagem, trabalha-se com o processo saúde-doença-cuidado, Que é complexo e carregado de valores, sentidos e significados, além do conhecimento morfofuncional.

Nesse sentido, tendo o cuidado como objeto da enfermagem no processo saúde-doença, a enfermagem brasileira a partir da década de 90 do último século, vem se dedicando na problematização teórico-prática e em experiências concretas sobre o Cuidado. Dentre várias autoras brasileiras destacamos alguns trabalhos ${ }^{(21-24)}$

Embora reconheçamos a importante contribuição da enfermagem brasileira em relação ao Cuidado ancorado nas diversas vertentes do conhecimento, vislumbra-se a necessidade de darmos continuidade a essa produção de conhecimento, articulada às experiências práticas/cotidianas da enfermagem. Entendemos Que tal articulação teoria-prática poderá se enriQuecer com a incorporação de outras correntes do conhecimento voltadas para a dimensão ontológica, existencial, dialógica e outras, pois o processo saúde-doença é sempre complexo carregado de valores éticos que expressa a singularidade do homem.

Outros pesQuisadores também vêm contribuindo para a reflexão sobre a importância do cuidado na produção de saúde. Dentre eles citamos Merhy ${ }^{(1)}$ Que vem discutindo a micropolítica do trabalho vivo em ato, e aí a singularidade e potência presentes no espaço intercessor em Que estão presentes trabalhadores e usuários em relação e em ação, sendo este o espaço onde é possível ocorrer o Cuidado. Mas para tal a dimensão profissional (conhecimentos e saberes) Que são tecnologias semi-duras têm Que estar aliadas à dimensão cuidadora Que é relacional, onde estão presentes as tecnologias leves Que permitem Que as intersubjetividades sejam contempladas.

José Ricardo de Carvalho Mesquita Ayres, médico sanitarista, vem construindo uma contribuição importante sobre o Cuidado, através de filósofos como Heidegger e Gadamer. Vamos nos basear nos seus construtos, pois estes podem enriquecer muito o nosso objeto de estudo, Que é o Cuidado. Nos dizeres de Ayres ${ }^{(25)}$ : "Embora a categoria Cuidado, na filosofia heideggeriana, não dizia respeito ao cuidar ou descuidar no sentido operativo do senso comum, e ainda menos numa perspectiva estritamente médica, adota-se o termo Cuidado como designação de uma atenção à saúde imediatamente interessada no sentido existencial da experiência do adoecimento, físico ou mental, e, por conseguinte, também das práticas de promoção, proteção ou recuperação da saúde".

Assim, só o êxito técnico não produz o Cuidado, ou seja, é necessário trazer para o momento do encontro entre profissional e usuários, o sentido existencial da experiência do adoecer e das práticas de saúde. Isto se dá por meio da escuta interessada e da dimensão dialógica do encontro Que propicia a fusão de horizontes de trabalhadores e usuários.

Além do sentido instrumental das ações de saúde e enfermagem, Que Ayres $^{(2)}$ denomina de êxito técnico, não nos esquecemos Que estes são históricos e sociais e como tais são saberes práticos. Ayres $^{(2)}$ considera Que o êxito técnico se refere ao sentido instrumental da ação, por exemplo, o uso da insulina na redução de riscos no diabetes. O sucesso prático se refere ao valor Que essa ação assume para indivíduos, populações, em razão das implicações simbólicas, relacionais e materiais dessas ações na vida cotidiana. No mesmo exemplo, o que significa estar com diabetes, para um indivíduo, para uma família e uma comunidade, cuidar da alimentação, tomar remédios, fazer controles periódicos, etc.

O mesmo autor traz ainda o conceito de projeto de felicidade, aquilo Que dá sentido existencial às demandas dos usuários e Que implica intersubjetividade, interação, compartilhamentos, aberturas. É neste sentido, um norte existencial. Nesta direção, compreendemos Que o conhecimento científico tem Que se abrir para a sabedoria prática e possibilitando o encontro dos horizontes normativos para produzir o Cuidado. A racionalidade prática das ações de saúde se assenta na linguagem e no diálogo. Para a hermenêutica, a dialética da linguagem é entendida como um modo de participação no mundo, e é sempre produzida entre sujeitos, e o valor da verdade prática se dá na fusão de horizontes, de um com o outro. O encontro terapêutico é amplo e rico de possibilidades de Cuidado.

\section{CONSIDERAÇÕES FINAIS}

O corpo de conhecimentos da enfermagem vem sendo construído, com predominância, na lógica da racionalidade científica empírica e analítica, Que lhe dá o "status" de disciplina/ciência e é ele Que vai instrumentalizar o Cuidado de enfermagem.

Se tomarmos o cuidado enQuanto procedimentos de enfermagem realizados para a promoção da saúde, prevenção da doença e reabilitação da saúde, este corpo de conhecimentos vai ter seu êxito técnico, pois produz o que se pretende. Mas se tomarmos o 
Cuidado como uma atenção à saúde, interessada no sentido existencial para re-fazer, restaurar o projeto de vida, e de uma vida boa, produzindo a fusão de horizontes do profissional, do usuário e também da comunidade, da gerência e mesmo das políticas de saúde, o arsenal técnico-científico de que a enfermagem dispõe não dará conta do sucesso prático.

O Cuidado não pode ser o produto de um saber exclusivamente instrumental provido do saber científico e tecnológico, pois, se assim ocorrer obteremos apenas uma parte da dimensão do cuidado, Que é o êxito técnico. Se Quisermos alcançar o sucesso prático para produzir cuidado, é necessária, além da dimensão instrumental, a produção de encontros e de diálogos entre sujeitos, sujeitos profissionais de saúde/serviços e sujeitos usuários e população. Na relação terapêutica, seja individual ou coletiva, o objeto não é o usuário, mas o Que se constrói a partir desses sujeitos em interação, numa fusão de horizontes ${ }^{(26)}$.

A enfermagem, Que tem como objeto o Cuidado, tem imensas possibilidades de aproximar os seus saberes instrumentais dos saberes práticos, na construção de intersubjetividades e fusão de horizontes para restaurar e restabelecer os projetos de vida e de uma vida boa. Cabe ressaltar ainda Que, o Cuidado eneuanto objeto da enfermagem, resultado da interação do êxito técnico e sucesso prático não apresentam apenas a dimensão micro, mas tem também o compromisso com valores do bem comum, pois a relação com o outro esta sempre presente. Assim, o sucesso prático pode oferecer elementos para melhor uso dos êxitos técnicos, como as estratégias de intervenção para o gerenciamento, para a organização de programas e para as políticas de saúde na dimensão social.

Portanto, o Cuidado permite fazer a mediação dialética entre as dimensões dos micros espaços de intervenção e dos macros espaços estruturais, permitindo a construção de práticas de saúde democráticas, convergentes com os direitos sociais. Depreendese, a partir dessas reflexões, a potência da enfermagem brasileira para protagonizar processos de transformação na sua prática, nas práticas de saúde, na educação, na pesquisa e nas políticas de saúde.

\section{REFERÊNCIAS}

I. Merhy EE. Saúde: cartografia do trabalho vivo. São Paulo: Hucitec; 2002.

2. Ayres IRCM. Hermenêutica e humanização das práticas de saúde. Ciênc Saúde Coletiva 2005; 10(3): 549-60.

3. Ministério da Saúde (BR). Política Nacional de Atenção Básica em Saúde. Portaria № 648, 28 de março de 2006. Brasília: Ministéiro da Saúde; 2006.

4. Almeida MCP, Rocha JSY. O saber de enfermagem e sua dimensão prática. São Paulo: Cortez: 1989.

5. Henderson V. Princípios básicos sobre cuidados de enfermagem. In: Conselho Internacional de Enfermagem. Rio de Ianeiro: ABEn; 1962.

6. Abdellah FG. Overview of nursing research 1955-1968. Part I. Nurs Res 1970; 19(1 Pt 1): 6-17.

7. Roy SC. Introduction to nursing: an adaptation model. Englewood Cliffs: Prentice-Hall; 1976.

8. Roy SC, Roberts SL. Theory construction in nursing: an adaptation model. Englewood: Prentice-Hall; 1981.

9. Levine ME. Introduction to clinical nursing. Filadélfia: F. A. Davis; 1973.

10. Brodt DE. A synergistic theory of nursing. Am I Nurs 1969; 69(8): 1674-6.

11. Rogers ME. An introduction to the theoretical bases of nursing. Filadélfia: F. A. Davis; 1970.

12. King EM. Illustrated manual of nursing techniques. Filadélfia: J.B. Lippincott; 1970.

13. Orem DE. Nursing concepts of practice. New York: McGrawHill; 1971.

14. Horta WA. Processo de enfermagem. São Paulo: EDUSP; 1979.

15. Carvalho EC, Bachion MM, Dalri MC, Jesus CAC. Obstáculos para a Implementação do Processo de Enfermagem no Brasil. Rev Enf UFPE 2007; I ( I): 95-9.

16. Conselho Federal de Enfermagem. Resolução COFEN 272/ 2002. Dispõe sobre Sistematização da Assistência de
Enfermagem - SAE. Rio de Janeiro: COFEN; 2002.

17. Farias IN, Nóbrega MML, Pérez VLAB, Coler MS. Diagnóstico de Enfermagem: uma abordagem conceitural e prática. João Pessoa: Santa Marta; 1990.

18. Cruz DALM. Contribuições do Diagnóstico de Enfermagem para a Autonomia da Enfermeira. In: Guedes MVC, Araújo TL organizadoras. O uso do Diagnóstico na Prática da Enfermagem. Brasília: Associação Brasileira de Enfermagem; 1997. p.51-8.

19. Almeida MCP, Palha PF, Domingos MMLN, Mishima SM, Vila TCS, Oba MDV. A Classificação Internacional da Prática de Enfermagem em Saúde Coletiva no Brasil CIPESC/ICN/ABEn e o Processo de Trabalho. In: Chianca TCM, Antunes MIM. A classificação internacional das práticas de enfermagem em saúde coletiva. Brasília: Associação Brasileira de Enfermagem; 1999. p. 46-56.

20. Napoleão AA, Chianca TCM, Carvalho EC, Dalri MCB. Análise da Produção Científica sobre a Classificação das Intervenções de Enfermagem (NIC) de 1980 a 2004. Rev Latino-am Enfermagem 2006; 14(4): 608-13.

21. Leopardi MT, Kirchhof AL, Capella BB, Pires DP, Faria EM, Ramos FRS, Vaz MRC. O Processo de Trabalho em Saúde: organização e subjetividade. Florianópolis: Papa-Livros; 1999.

22. Lima MADS, Almeida MCP. O trabalho de enfermagem na produção de cuidados de saúde no modelo clínico. Revista Gaúcha de Enfermagem 1999; 20(supl).

23. Figueiredo NMA, Porto IS, Santos I, Cardoso FCA, Souza FS. Ensinando Alunas (os) a Sentir: areuitetando uma semiologia da expressão para cuidar - os sentidos e os cuidados. Rev Enfermagem UERJ 2001; 9: 217-24.

24. Waldow VR. O Cuidado na Saúde: as elações entre o eu, o outro e o cosmos. Petrópolis: Vozes; 2004.

25. Ayres IRCM. O cuidado, os modos de ser (do) humano e as práticas de saúde. Rev Saúde Sociedade 2004; 13(3): 16-29.

26. Ayres IRCM. Uma concepção hermenêutica de saúde. Rev Saúde Coletiva 2007;17(1): 43-62. 